\title{
Infodemia y COVID-19. Evolución y viralización de informaciones falsas en España
}

\section{Infodemia and COVID-19. Evolution and viralization of false information in Spain}

José Manuel Sánchez-Duartea , Raúl Magallón Rosab

a Departamento de Derecho Público I y Ciencia Política, Facultad de Derecho y Ciencias Sociales, Universidad Rey Juan Carlos,

España

b Departamento de Comunicación, Universidad Carlos III de Madrid, España

\section{Resumen}

Introducción: La verificación de la información supone un reto democrático especialmente en periodos de excepcionalidad y crisis. Objetivo: Analizar el tipo de bulos identificados durante la pandemia del COVID-19 según su temática, fecha de verificación, canales de distribución, intencionalidad, viralización en otros países e identificación de sus promotores. Metodología: Se han analizado 166 bulos reportados por la organización Maldita.es a la plataforma de verificación colaborativa LatamChequea desde el 14 de febrero al 15 abril de 2020. Resultados: Entre los principales resultados destaca la alta presencia de bulos en torno a contagios y medidas de prevención. Conclusiones: El aumento de la desinformación se desarrolla en paralelo a la evolución de la pandemia, destaca la viralización de los bulos mediante redes sociales y servicios de mensajería instantánea, además de su alcance internacional y la alta dificultad para identificar a sus promotores.

Palabras clave: desinformación; redes sociales; WhatsApp, COVID-19; coronavirus; fact-checking; bulos; España; medios de comunicación.

\begin{abstract}
Introduction: Verifying information is a democratic challenge, especially in periods of exceptionality and crisis. Objective: To analyze the type of hoaxes identified during the COVID-19 pandemic according to their topic, verification date, distribution channels, viralization in other countries and identification of their promoters. Methodology: 166 hoaxes reported by the organization Maldita.es to the collaborative verification platform LatamChequea from February 14 to April 15, 2020 have been analyzed. Results: Among the main results, we draw attention to the high presence of misinformation around infections and preventive measures. Conclusions: Misinformation and disinformation increases as the pandemic sets in and a series of containment measures are taken, we hightlight the viralization of hoaxes through social networks and instant messaging services, in addition to its international reach and the high difficulty in identifying its promoters.

Keywords: disinformation; social networks; WhatsApp; COVID-19; coronavirus; fact-checking; hoaxes, Spain; media.
\end{abstract}




\section{Introducción}

$\square$ nlaactualidad, vivimosunmomento de normalización de todos los procesos de desinformación en la esfera pública. La Organización Mundial de la Salud había advertido de que un exceso de información podía dificultar la tarea de encontrar información fiable sobre la pandemia de COVID-19 (World Health Organization [WHO], 2020). Además, las informaciones falsas que tienen, en apariencia, carácter científico se propagan fácilmente por redes sociales porque el consumidor de información no puede contrastarlas fácilmente ni sabe a qué fuentes fiables acudir (Lytvynenko, 2020).

En este contexto, la mediatización extrema generada por el confinamiento reforzó la idea de que el miedo y la desinformación están estrechamente vinculados. Cuanto menos se puede ver y comprobar la realidad físicamente, más dudas aparecen sobre el contenido cierto de los acontecimientos. El miedo es una pasión etológica que aparece por la inclusión de un sujeto u objeto extraño en nuestro territorio y, en este caso, tener la sensación de falta de control sobre lo que sucede permite que se extiendan aún más este tipo de pasiones.

En este sentido se produjeron varios fenómenos que siguieron alimentando el escenario de desinformación. En primer lugar, la evolución de la pandemia a nivel global hizo crecer la desconfianza hacia las cifras oficiales ofrecidas por China (Corera, 2020). En segundo lugar, esa opacidad fue esgrimida como argumento para justificar la relación entre causalidad y responsabilidad -desarrollándose una narrativa en aquellos países que empezaban a verse más afectados por las muertes, por la economía, por la mala gestión, etc.- (Reicher, 2020). En tercer y último lugar, están los factores geoestratégicos a corto y largo plazo. Con tres actores principales: la propaganda china como suministradora de materiales sanitarios y como el nuevo actor de paz global, el papel del dólar y de EEUU como primera fuerza económica y geopolítica, y el componente de desestabilización permanente que siempre subyace en la propaganda en torno a Rusia.

Desde esta perspectiva, el gobierno ruso considera que las herramientas no militares pueden ser potencialmente más poderosas que las herramientas militares y para ello se han de utilizar instrumentos como la guerra de información, la manipulación cultural o el hacktivismo en las redes sociales para lograr objetivos de política exterior sin el uso de la fuerza directa (Seely, 2018).

La literatura académica más reciente ya estudia de forma profusa los procedimientos de las estrategias de desinformación a escala global. Autores como Allcott \& Gentzkow (2017), Shao et al. (2018), Faris et al. (2017), Benkler et al. (2017), Wardle \& Derakhshan (2017), Pennycook et al. (2018), Vosoughi et al. (2018), Gorodnichenko et al. (2018), Nyhan \& Reifler (2012), Humprecht $(2018,2019)$ y Humprecht et al. (2020) han elaborado un marco teórico desde el cual se puede seguir investigando y reflexionando sobre estos procedimientos.

La aproximación al fenómeno de la desinformación ha ido volviéndose cada vez más glocal, frente a las primeras investigaciones que hacian referencia principalmente a las elecciones estadounidenses y al contexto anglosajón. Al respecto, Innerarity (2020) señala que la crisis del coronavirus es "un acontecimiento pandemocrático, como todos los riesgos globales. Se da la paradoja de que un riesgo que nos iguala a todos revela al mismo tiempo lo desiguales que somos, provoca otras desigualdades y pone a prueba nuestras democracias".

De igual modo, las investigaciones se han vuelto cada vez más especializadas incorporando nuevas metodologías (Vázquez-Herrero et al., 2019) y actores que intentan combatir y describir la actualización del software de la desinformación que se va desarrollando en distintos ámbitos que a priori poco tienen en común, como son la política o la ciencia (Molina-Cañabate \& Magallón-Rosa, 2020). Al respecto, uno de los problemas que se plantean en la lucha contra la desinformación científica es que los consumidores habituales de noticias de este tipo resultan menos comprometidos en la difusión de la ciencia y más propensos a comentar en las páginas de conspiración (Bessi et al., 2015).

Asimismo, se pone de manifiesto la importancia no sólo de la profesión periodística (Mayo-Cubero, 2019), sino de entidades verificadoras de información, altamente especializadas en estos procesos (Humprecht, 2019). A principios de abril de 2020 se estimaba que había 237 equipos activos dedicados al fact-checking en todo el mundo -además de 92 inactivos-; de los cuales sólo llegaban a 71 las organizaciones periodísticas de fact-checking activas que habían firmado el código de principios de la International Fact Checking Network (IFCN). En España, forman parte Newtral, Efe Verifica, AFP y Maldita.

El código de la IFCN fue lanzado en septiembre de 2016 y promueve la excelencia en la comprobación de hechos de forma no partidista y transparente. En enero 
de 2017, la IFCN presentó el procedimiento de solicitud de ingreso en la misma tras el anuncio de Facebook de que ser firmante de este código era una condición mínima para ser aceptado como un verificador de datos en su red social.

Entre los principios de la IFCN destacan el compromiso con el no partidismo y la equidad, el compromiso con la transparencia de las fuentes, de la transparencia en la financiación de la organización, la transparencia metodológica (para seleccionar, investigar, escribir, editar, publicar y corregir los casos escogidos para la verificación de hechos) y con las correcciones abiertas y honestas (incluidas las versiones corregidas). En medio de la pandemia, el IFCN anunció que iba a fortalecer sus estándares para generar confianza (IFCN, 2020b). El nuevo código incluye nuevas reglas sobre quién puede ser firmante, prohíbe los medios controlados por el estado, exige que los firmantes se centren principalmente en cuestiones de interés público, requiere un período de prueba más largo y desarrolla el principio de no partidismo. Desde esta perspectiva, la evolución de las iniciativas de fact-checking han seguido un recorrido diferente dependiendo del país, del desarrollo mediático, de los usos digitales y del tipo de verificación (Clayton et al., 2019).

Si utilizamos las redes sociales para el análisis de rumores y el estudio de los procesos de circulación de desinformación y de propaganda, parece pertinente pensar que podemos servirnos de los verificadores de hechos para hacer una tipología de los diferentes tipos de bulos que se desmienten, así como de la estructura que tienen dependiendo de la temática (MagallónRosa, 2018a).

Centrándonos en nuestro objeto de estudio, infodemia y COVID-19, destaca la investigación del Reuters Institute dirigida por Rasmus K. Nielsen que señalaba que es menos probable que personas con bajos niveles de educación digan que dependen de los medios para obtener noticias e información sobre el coronavirus y más probable que confíen en las redes sociales y las aplicaciones de mensajería (Nielsen et al., 2020)1. Desde este punto de vista, los primeros estudios sobre desinformación y COVID-19 señalan que empujar a las personas a pensar sobre la precisión de un contenido es una forma simple de mejorar las opciones sobre qué

$\overline{1}$ El estudio se realizó en Argentina, Alemania, Corea del Sur, España, Reino Unido y EE. UU. compartir en las redes sociales. Por ejemplo, valorar la precisión de los titulares duplica el nivel de discernimiento compartido entre los titulares verdaderos y falsos en los experimentos de su trabajo (Pennycook et al., 2020).

\section{LatamChequea-Coronavirus}

En 2014, coordinado por el medio digital argentino Chequeado, se llevó a cabo el primer encuentro regional de innovación, periodismo y verificación de datos de América Latina. En años sucesivos se repitieron estas jornadas con el fin de reunir a organizaciones, editores y directores de medios y periodistas para compartir experiencias y metodologías en torno a la verificación de datos y crear una red de verificadores en la región.

A partir de esta iniciativa, el 2 de abril de 2020, 21 organizaciones de fact-checking ${ }^{2}$ de 14 países diferentes lanzan el proyecto colaborativo LatamChequeaCoronavirus para trabajar juntas en la verificación de contenidos sospechosos en torno a la pandemia -con fecha 15 de abril de 2020 eran 27 las organizaciones implicadas-. Como recogen en su web: "Frente a la 'infodemia', la difusión de rumores y noticias contenidos falsos, los chequeadores de Latinoamérica nos aliamos para compartir la información que producimos $y$, al unir esfuerzos, brindar mejor información a nuestras comunidades. Las desinformaciones que circulan en muchos casos son las mismas en distintos países y poder contar con el trabajo de otros ayuda a desmentir más rápidamente las falsedades y evitar su propagación" (LatamChequea, 2020).

La importancia de emplear los datos de esta plataforma para la investigación radica en que su base de datos está realizada a partir de las recomendaciones y el código de buenas prácticas elaborado por la IFCN del Instituto Poynter. De igual modo, LatamChequea-Coronavirus desarrolla el proyecto en español y portugués de este centro a nivel mundial -en el que a mediados de mayo de 2020 están agrupados más de 70 países- (IFCN, 2020).

Hasta ahora, muchas de estas iniciativas colaborativas se habían centrado en exclusiva en periodos electorales a nivel local. Así, LatamChequea-Coronavirus replica

$\overline{2}$ Las organizaciones participantes en principio eran: AFP Factual; Salud con Lupa, Estadao Verifica y Lupa de Brasil, Bolivia Verifica, Mala Espina Check de Chile, La Silla Vacía y ColombiaCheck de Colombia, La Nación y la Voz de Guanacaste de Costa Rica, Periodismo de Barrio y El Toque de Cuba, Ecuador Chequea y GK de Ecuador, Maldita y Newtral de España, Agencia Ocote de Guatemala, Animal Político y Verificado de México, Despacho 505 de Nicaragua, El Surtidor de Paraguay, OjoPúblico y Convoca de Perú, PoletikaRD de República Dominicana, UyCheck de Uruguay y Cotejo.info y Efecto Cocuyo de Venezuela. 
en parte, y de una manera transnacional, iniciativas de fact-checking colaborativo en distintos países: CrossCheck en Francia, Electionland en Estados Unidos, Verificado en México, Comprova en Brasil, Checkpoint en India, Comprobado en España, Reverso en Argentina o Verificado en Uruguay. Estas iniciativas permitieron establecer tipologías de bulos electorales funcionando como alertas tempranas de desinformación. El estudio de variables como su origen en el proceso de distribución, su tipología, la temática o la víctima (persona o institución perjudicada por la difusión del rumor) resultaron fundamentales para conocer los procesos de circulación de la desinformación, pero también para dar respuestas coordinadas y eficaces.

En relación a los datos sobre España presentes en LatamChequea-Coronavirus, estaban los aportados por Maldita.es y por Newtral. Maldita.es es una organización que tiene como objetivo "monitorizar y controlar el discurso político y promover la transparencia en las instituciones públicas y privadas". En el mes de marzo de 2020 , en plena explosión de la pandemia, llegaron a más de 10 millones de visitas solo a través de su sitio web, centralizando toda la información sobre la COVID-19 en un apartado especial con más de 300 piezas, resolviendo dudas y consultas de su comunidad y difundiendo información científica -utilizando fuentes oficiales y expertos para resolverlas (Maldita, 2020).

Por su parte, Newtral es un proyecto empresarial de contenido audiovisual fundado en enero de 2018 y centrado en tres áreas de negocio principalmente: la producción de programas de televisión y nuevas narrativas en redes sociales, la innovación en el periodismo a través del fact-checking y la investigación basada en los protocolos de inteligencia artificial.

La evolución de la pandemia de COVID-19 a nivel global y la ampliación de los periodos de confinamiento están redefiniendo la manera que tenemos de acercarnos a la información (Jurkowitz \& Mitchell, 2020), pero también la forma en la que la desinformación circula (Brennen et al., 2020). Por ello, el objetivo de esta investigación se centra en analizar los datos sobre España presentes en el proyecto colaborativo LatamChequea durante la pandemia del COVID-19.

\section{Método}

Con el fin de tener una comprensión más exhaustiva del objeto de estudio se plantea un diseño de investigación mixto. Así, el paradigma cuantitativo se desarrolla con la medición de las variables: tipología, evolución, origen, intencionalidad, viralización e identificación de promotores. La investigación se complementa con una entrevista en profundidad semiestructurada a un informante especial con una posición privilegiada en la organización objeto de análisis. De esta manera se proporciona una vía interpretativista y cualitativa a la investigación (Corbetta, 2010)

El desarrollo de este proyecto tiene como marco de trabajo una idea recurrente en diferentes estudios sobre desinformación en los que se expresa la necesidad de crear y diseñar una hemeroteca de bulos y valorar la posibilidad de crear un archivo digital con rumores previamente desmentidos (Magallón-Rosa, 2018b).

Elección de la muestra, variables y técnica de análisis

Para la investigación se seleccionaron todas las informaciones pertenecientes a España y aportadas por Maldita (168) y Newtral (46) a LatamChequea-Coronavirus entre el 14 de febrero y el 15 de abril de 2020. Esta selección, hecha por el equipo de Chequeado a partir de las verificaciones en español y portugués realizadas para la CoronaVirusFacts Alliance, abarcaba el mes previo a la declaración del Estado de Alarma por parte del Gobierno de España y un mes con la vigencia de dicho estado. La selección responde a la necesidad de identificar los tipos de bulos y su funcionamiento en dos periodos de tiempo con diferencias de contexto sustanciales.

Tras un primer análisis se decidió centrar la investigación en las piezas aportadas por Maldita, ya que los datos de la organización Newtral abarcaban solo el mes con el Estado de Alarma vigente. De esta manera se seleccionaron 166 piezas $^{3}$ que fueron codificadas en las siguientes variables. Aunque muchas de estas clasificaciones estaban presentes en la base de datos de LatamChequea-Coronavirus sus categorías fueran recodificadas, ajustándolas a los objetivos de nuestra investigación.

1) Tipología de bulos: contagios, estado y evolución de la pandemia; formas de prevención y curas; medidas (públicas y privadas) adoptadas en la lucha contra la pandemia y para paliar sus efectos y otros (tabla 1).

3 De las 168 piezas, 166 eran bulos y sólo 2 informaciones veraces. 
Tabla 1. Tipología de bulos.

\begin{tabular}{|c|l|}
\hline Tipología de bulos & \multicolumn{1}{|c|}{ Ejemplos } \\
\hline Contagios & $\begin{array}{l}\text { Estado y evolución de los contagiados, número, } \\
\text { zonas de contagio, situaciones concretas de } \\
\text { localidades con focos contagiosos, evolución } \\
\text { de la enfermedad, situaciones de caos, } \\
\text { características y síntomas. }\end{array}$ \\
\hline Prevención & $\begin{array}{l}\text { Formas y métodos de prevención, curas, } \\
\text { remedios, etc. }\end{array}$ \\
\hline pandemia (públicas y privadas) y para paliar & $\begin{array}{l}\text { Cierre de espacios, controles y restricciones, } \\
\text { órdenes gubernamentales y de partidos } \\
\text { políticos, prohibiciones, presencia del ejército, } \\
\text { acciones desplegadas por empresas, etc. }\end{array}$ \\
\hline Otros & $\begin{array}{l}\text { Cuestiones relacionadas con la seguridad } \\
\text { (asaltos a domicilios, seguridad de aplicaciones } \\
\text { informáticas, estafas (phishing), teorías acerca } \\
\text { del origen del virus, predicciones sobre la } \\
\text { llegada de la pandemia, informaciones sobre } \\
\text { grupos de población específicos, etc. }\end{array}$ \\
\hline
\end{tabular}

2) Fecha de verificación: Antes del Estado de Alarma (del 14 de febrero al 14 de marzo de 2020) y durante el Estado de Alarma (del 15 de marzo al 15 de abril de 2020)

3) Origen y canales de distribución del bulo: redes sociales, mensajería instantánea o medios de comunicación.

4) Intencionalidad del bulo: Desmentir o ampliar información.

5) Viralización en otros países: Viralizado o no viralizado.

6) Identificación de los promotores de bulo: Identificado o no identificado.

Con la intención de complementar este análisis se realizó una entrevista en profundidad a Ximena Villagrán, responsable de proyectos e impacto en Maldita. A partir de la noción de informantes especiales: personas con información directa y relevante para los propósitos de la investigación y una posición única en la organización a investigar (Vallés, 1999) se hizo una entrevista semiestructurada. La entrevista se distribuyó en dos bloques temáticos diferenciados. En el primero, centrado en la metodología de trabajo de Maldita y su relación con LatamChequea-Coronavirus, se preguntaron cuestiones relacionadas con los procedimientos de verificación, los patrones de relación organización-proyecto y las herramientas y dispositivos empleados. En el segundo bloque se abordaron preguntas centradas en los bulos, tipologías y comportamiento a lo largo de la pandemia. La información derivada de esta investigación ha servido para articular el análisis y la discusión de la investigación, aportar claridad interpretativa a los resultados y dotar de una mayor fortaleza a las variables planteadas.

\section{Resultados}

El barómetro especial de abril de 2020 del Centro de Investigaciones Sociológicas (CIS) situaba el COVID-19 como el principal problema en España (CIS, 2020). La inclusión de un tema dentro de la agenda social aumenta la demanda informativa sobre él, más si este irrumpe con rapidez y se desarrolla en un marco de incertidumbre. Como indica el informe sobre "Consumo de información durante el confinamiento por coronavirus", el $78 \%$ de la ciudadanía se informó más que antes de la pandemia sobre el virus y el $52 \%$ lo hacía tres o más veces al día - cuando antes de la crisis sólo lo hacía el 28\%- (Masip et al. 2020). En ese mismo informe se recoge que más del $80 \%$ de las personas encuestadas admitían haber recibido noticias falsas o de dudosa veracidad sobre la pandemia.

En esta línea, el Reuters Institute de la Universidad de Oxford advirtió del aumento un $900 \%$ de las noticias 
verificadas en inglés a partir de bulos entre enero y marzo de 2020 siendo en su mayoría (59\%) informaciones erróneas que reconfiguran, tuercen y reelaboran la información existente y un $38 \%$ noticias completamente elaboradas desde cero (Brennen et alt, 2020).

Las piezas reportadas por Maldita a la plataforma LatamChequea son mayoritariamente bulos. El 98\% de las verificaciones realizadas eran informaciones falsas. Un porcentaje tan elevado advierte que el modo de funcionar de los fact-checkers se apoya en una comunidad de usuarios muy activos con una serie de capacidades elevadas para prevenir y detectar bulos. Aquellas informaciones reportadas a las plataformas de verificación por esta comunidad, con habilidades y prácticas previas, tienen mucha probabilidad de ser bulos.

En relación a los temas en los que se centraban los 166 bulos analizados se aprecia una distribución homogénea por categorías con porcentajes similares. La diversidad temática combina informaciones sobre los contagios, el estado y la evolución de la pandemia, las formas de prevención y curas, y las medidas adoptadas para paliar la crisis. Sólo la categoría de otros, con cierta diversidad interna de cuestiones (seguridad, teorías y conspiraciones sobre el origen del virus) despuntaba aunque mínimamente (tabla 2).

Sobre la distribución de los bulos verificados durante el periodo de investigación, se identifica un aumento significativo de la desinformación a medida que la pandemia se iba desarrollando. En el mes previo al decreto de Estado de Alarma sólo se reportaron un $32,5 \%$ de los bulos pasando a $67,5 \%$ en el mes posterior (Tabla 3). Varios son los factores que podrían explicar este aumento. En periodos de alta intensidad informativa la ciudadanía precisa de manera urgente ampliar sus datos y obtener detalles sobre los hechos narrados en las noticias. Más cuando el conocimiento sobre la realidad es, casi en exclusiva, mediado a través de medios de comunicación convencionales y ecosistemas informativos en red. A esto hay que añadir la rapidez en el desarrollo de los acontecimientos y el desconocimiento sobre los sucesos que estaban sucediendo, propiciando las condiciones perfectas para la creación y difusión de desinformaciones.

Cabe destacar cómo aquellas desinformaciones centradas en la prevención y medidas aparecen indistintamente en ambos periodos y de manera transversal. Pese a ello, hay que subrayar una mayor presencia de los bulos sobre contagios en la primera etapa de la crisis y una diversificación de las desinformaciones entre el 15 de marzo y el 15 de abril. Así, este tipo de bulos dieron paso a piezas centradas en el origen de la pandemia, cuestiones relacionadas con la seguridad (estafas, fallos en aplicaciones informáticas, asaltos a domicilio) o informaciones focalizadas en determinados colectivos de población (migrantes) englobados en la categoría otros (tabla 3).

Tabla 2. Distribución de los tipos de bulos ( $\mathrm{N}=166)$.

\begin{tabular}{|c|c|}
\hline & Porcentajes \\
\hline Contagios & $21,7 \%$ \\
\hline Prevención & $24,7 \%$ \\
\hline Medidas & $24,7 \%$ \\
\hline Otros & $28,9 \%$ \\
\hline Total & $100 \%$ \\
\hline
\end{tabular}


Tabla 3. Periodo de aparición de los bulos ( $\mathrm{N}=166)$.

\begin{tabular}{|c|c|}
\hline & Porcentajes \\
\hline $\begin{array}{c}\text { Antes del Estado de Alarma (Del 14 de febrero al 14 de } \\
\text { marzo) }\end{array}$ & $32,5 \%$ \\
\hline $\begin{array}{c}\text { Durante del Estado de Alarma (Del 15 de marzo al 15 de } \\
\text { abril) }\end{array}$ & $67,5 \%$ \\
\hline Total & $100 \%$ \\
\hline
\end{tabular}

Los canales de información más relevantes para vehicular bulos fueron las redes sociales $(36,1 \%)$ y las aplicaciones de mensajería instantánea (36,1\%) (Tabla 4). En el caso de las redes, resaltan Facebook y Twitter, y en menor medida YouTube e Instagram. Sobre las aplicaciones de mensajería, WhatsApp aparece como hegemónica. Si lo comparamos con el resto de categorías, ambos canales (redes y mensajería) se presentan como el ecosistema natural de circulación y difusión de los bulos. Esta característica está corroborada por estudios previos (Tárdáguila, Benevenuto \& Ortellado, 2018) y se corresponde además con los nuevos patrones de consumo de información (tabla 4).

En relación a la intencionalidad de los bulos destacan varias cuestiones (Tabla 5). La primera de ellas es la baja presencia de piezas centradas en la verificación de figuras públicas o de medios de comunicación (apenas un $5 \%$ del total). El grueso de la muestra se reparte entre las desinformaciones que explican rasgos de la pandemia $(26,5 \%)$ y, en especial, en desinformaciones virales que desmienten y reconfiguran noticias previas $(68,7 \%)$.

Las piezas de desinformación sobre contagios y prevención se presentaban como mensajes que ampliaban o explicaban con detalle cuestiones relacionadas con esos ámbitos. Los desmentidos de estos bulos respondían a preguntas como: “¿Qué se sabe acerca de?”. Y a las que, en palabras de la responsable de proyectos e impacto de Maldita, se ha añadido la cuestión: "¿Qué evidencia científica se tiene sobre?" (tabla 5).

Tabla 4. Canales de distribución de los bulos ( $N=166)$.

\begin{tabular}{|c|c|}
\hline & Porcentajes \\
\hline Redes sociales & $36,1 \%$ \\
\hline Mensajería instantánea & $36,1 \%$ \\
\hline Medios de comunicación & $8,4 \%$ \\
\hline Origen desconocido & $13,3 \%$ \\
\hline Otros & $6 \%$ \\
\hline Total & $100 \%$ \\
\hline
\end{tabular}


Tabla 5. Intencionalidad de bulos ( $\mathrm{N}=166)$.

\begin{tabular}{|c|c|}
\hline & Porcentajes \\
\hline Desinformación viral & $68,7 \%$ \\
\hline Explicadores & $26,5 \%$ \\
\hline Verificación a figuras públicas & $2,4 \%$ \\
\hline Verificación a medios & $2,4 \%$ \\
\hline Total & $100 \%$ \\
\hline
\end{tabular}

Otro de los rasgos destacables de la muestra analizada indica cierto carácter transnacional de los bulos (Tabla 6). Si bien el porcentaje de desinformaciones que fueron localizadas en otros países suponía sólo el $20 \%$ se identifica una tendencia cada vez más común. Los promotores de bulos tienen la capacidad de readaptar cualquier tipo de información a un contexto local. Esta técnica permite que la ciudadanía muestra una mayor atención a las noticias que les llegan por diferentes canales al sentir su contenido como más cercano y cruzando fronteras.

Como indica el informe del Reuters Institute de la Universidad de Oxford, citado con anterioridad, casi el $60 \%$ de los bulos identificados fueron noticias reconstruidas 0 recontextualizadas. Así, cualquier tema sin importar su condición o temática puede ser adaptado a localidades 0 áreas geográficas locales aunque su punto de partida esté identificado en otros países (tabla 6).

La última característica de los bulos presentes en LatanChequea-Coronavirus se centra la identificación de sus promotores. El anonimato constituye una de las señas de identidad en el funcionamiento de las desinformaciones (Tabla 7). Casi en el $75 \%$ de las piezas verificadas no se determina su origen, frente al $25 \%$ en el que los promotores fueron identificados. La rápida difusión y las posibilidades de reenvío y publicación en plataformas de mensajería y redes sociales contribuyen a que la autoría de los bulos quede difuminada (tabla 7).

Tabla 6. Viralización en otros países ( $\mathrm{N}=166)$.

\begin{tabular}{|c|c|}
\hline & Porcentajes \\
\hline Viralizado & $19,9 \%$ \\
\hline No viralizado & $80,1 \%$ \\
\hline Total & $100 \%$ \\
\hline
\end{tabular}

Tabla 7. Identificación de promotores (N=166).

\begin{tabular}{|c|c|}
\hline & Porcentajes \\
\hline Identificado & $25,3 \%$ \\
\hline Desconocidos & $74,7 \%$ \\
\hline Total & $100 \%$ \\
\hline
\end{tabular}




\section{Discusión y conclusiones}

La iniciativa LatamChequea-Coronavirus destaca por tratarse de un proyecto colaborativo transnacional, por establecer una metodología común para monitorizar la desinformación y por la novedad de poner los datos obtenidos en formato abierto para que investigadores puedan realizar estudios en tiempo real.

En este contexto, la presente investigación resalta como los bulos recogidos en la plataforma tuvieron una amplia variedad temática abarcando cuestiones relacionadas con los contagios, las formas de prevención, las medidas públicas y privadas adoptadas y otras cuestiones relacionadas con la seguridad o el origen de la pandemia. De igual modo, el aumento de la desinformación fue parejo al desarrollo de la crisis y a las medidas tomadas por los gobiernos para aminorarla vehiculándose de un modo masivo por redes sociales y plataformas de mensajería instantánea como WhatsApp.

Los resultados confirman que la desinformación aumenta a medida que se va instalando la pandemia y se van tomando una serie de medidas de contención. Más de dos tercios de los bulos monitorizados aparecen en el radar del fact-checker Maldita a partir del 15 de marzo de 2020. También los bulos adoptaron formatos novedosos. A las desinformaciones virales "convencionales" se le unieron significativamente los "explicadores": piezas centradas en cómo prevenir o actuar ante el coronavirus.

Por último, cabe destacar dos características importantes. Se advierte una tendencia a la internacionalización de los bulos localizando piezas generadas en España en otros países como EEUU, Argentina, Colombia o México. Esto rompe tendencias de desinformación política que establecen narrativas globales, pero casos concretos locales.

De igual modo, y mayoritariamente, en los bulos sigue prevaleciendo el anonimato y la dificultad para identificar su autoría. En cualquier caso, queremos destacar que en un $25,3 \%$ de la desinformación monitorizada por la organización periodística Maldita se llega a identificar el origen o el amplificador del bulo. Sin embargo, es importante subrayar que no hay una relación entre el origen y el tipo de bulo. Es decir, el tipo de bulo no se relaciona con la fuente de desinformación por lo que variables como la actualidad mediática y política pueden ser un factor para analizar en futuras investigaciones. Desde este punto de vista, estudios posteriores nos podrán dar mayor información sobre cómo afectan los ciclos de actualidad a la creación de bulos, por ejemplo, vinculando las verificaciones publicadas con las búsquedas en Google Trends. Al respecto, se plantean dos hipótesis: un aumento en las búsquedas de Google puede conllevar un aumento sobre los bulos sobre esas búsquedas -por ejemplo, en vacunas-, o bien que las búsquedas aumenten por un periodo de publicación creciente de verificaciones e informaciones de actualidad relacionadas con la problemática.

Para finalizar, es necesario destacar que la tipología de desinformaciones tiene una relación con su intencionalidad y con los canales por los que son distribuidas. Los bulos "explicadores" encontraron en la pandemia un terreno fértil para difundir desinformaciones sobre contagios y sobre cómo prevenirlos. También encontraron vías fructíferas, rápidas e incontrolables para su distribución a través de redes sociales y grupos de WhatsApp.

\section{Referencias bibliográficas}

Allcott, H., \& Gentzkow, M. (2017). Social media and fake news in the 2016 election. Journal of Economic Perspectives, 31(2), 211-236.

Benkler. Y., Faris, R., Roberts, H., \& Zuckerman, E. (2017). Study: Breitbart-led right-wing media ecosystem altered broader media agenda. Columbia Journalism Review. Recuperado de https://www.cjr. org/analysis/breitbart-media-trump-harvard-study.php

Bessi, A., Coletto, M., Davidescu, G., Scala, A., Caldarelli, G., \& Quattrociocchi, W. (2015). Science vs Conspiracy: Collective Narratives in the Age of Misinformation. PLOS ONE, 10(2), 1-17. doi: 10.1371/journal.pone.0118093

Brennen, J., Simon, F., Howard, P., \& Rasmus, K. (2020). Types, Sources, and Claims of COVID-19 Misinformation. Oxford: Reuters Institute for the Study of Journalism. Recuperado el 20 de abril 2020 de https://reutersinstitute.politics.ox.ac.uk/typessources-and-claims-covid-19-misinformation

CIS (2020). Barómetro Especial de Abril 2020. Estudio 3279. Centro de Investigaciones Sociológicas. Recuperado el 19 de abril de 2020 de http://datos.cis.es/pdf/Es3279mar_A.pdf

Clayton, K., Blair, S., Busam, J. A., Forstner, S., Glance, J., Green, G.,... Nyhan, B. (2019). Real Solutions for fake news? Measuring the Effectiveness of General Warnings and Fact-Check Tags in Reducing Belief in 
False Stories on Social Media. Political Behaviour, 40, 1-24. doi: 10.1007/s11109-019-09533-0

Corbetta, P. (2010). Metodología y técnicas de investigación social. Madrid: McGraw Hill.

Corera, G. (2020, 23 de abril). Coronavirus: Europe 'wary of confronting China over deaths. $B B C$. Recuperado de: https://www.bbc.com/news/worldasia-china-52404612

Faris, R., Roberts, H., Etling, B., Bourassa, N., Zuckerman, E., \& Benkler, Y. (2017). Partisanship, Propaganda, and Disinformation: Online Media and the 2016 U.S. Presidential Election. Berkman Klein Center for Internet \& Society, 6, 1-140. Recuperado de https://ssrn.com/abstract=3019414

Gorodnichenko, Y., Pham, T., \& Talavera, O. (2018). Social Media, Sentiment and Public Opinions: Evidence from \#Brexit and \#USElection. NBER Working Paper, 24631. Recuperado de http://papers.nber.org/ tmp/24427-w24631.pdf

Humprecht, E., Esser, F., \& Van Aelst, P. (2020). Resilience to Online Disinformation: A Framework for Cross-National Comparative Research. The International Journal of Press/Politics, 25(3), 1-24. doi: 10.1177/1940161219900126

Humprecht, E. (2019). How Do They Debunk "fake news"? A Cross-National Comparison of Transparency in Fact Checks. Digital Journalism, 8(3), 310-327. doi: 10.1080/21670811.2019.1691031

Humprecht, E. (2018). Where 'fake news' flourishes: a comparison across four Western democracies. Information, Communication \& Society, 22(13), 1973-1988. doi: 10.1080/1369118X.2018.1474241

IFCN. (2020a). The CoronaVirusFacts/DatosCoronaVirus Alliance Database. International Fact-checking Network. Recuperado de https://www.poynter.org/ ifcn-covid-19-misinformation/

IFCN. (2020b). IFCN strengthens its standards to build trust in global fact-checking. International Factchecking Network. Recuperado de https://www. poynter.org/fact-checking/2020/ifcn-strengthens-its- standards-to-build-trust-in-global-fact-checking/

Innerarity, D. (2020). Pandemocracia. Barcelona, España: Galaxia Gutenberg.

Jurkowitz, M., \& Mitchell, A. (2020). Cable TV and COVID-19: How Americans Perceive the Outbreak and View Media Coverage Differ by Main News Source. Pew Research Center. Recuperado de https:// www.journalism.org/2020/04/01/cable-tv-and-covid19-how-americans-perceive-the-Outbreak-and-viewmedia-coverage-differ-by-main-news-source

LatamChequea. (2020). Información chequeada sobre el Coronavirus. Recuperado de https://www. chequeado.com/latamcoronavirus/

Lytvynenko, J. (2020, 24 de marzo). Here's a Running List of the Latest Hoaxes Spreading about the Coronavirus, BuzzFeed News. Recuperado de https://www.buzzfeednews.com/ article/janelytvynenko/coronavirus-fake-newsdisinformation-rumors-hoaxes

Magallón-Rosa, R. (2018a). Nuevos formatos de verificación. El caso de Maldito Bulo en Twitter. Sphera Publica, 1(18), 41-65.

Magallón-Rosa, R. (2018b). La biblioteca digital sobre Donald Trump. Fact-checking frente a fake news. Estudios sobre el Mensaje Periodístico, 24(1), 273-282. doi: 10.5209/ESMP.59949

Maldita.es. (2020). Especial coronavirus. Recuperado de https://maldita.es/coronavirus/

Mayo-Cubero, M. (2019). Uso de las redes sociales en la cobertura periodística de crisis, desastres y emergencias en España. Revista Española de Comunicación en Salud, Suplemento 1, 43-54. doi: http://dx.doi.org/10.20318/recs.2019.4428

Masip, P., Almenar, E., Aran, S., Capilla, P., Puertas, D., Ruiz, ... Zilles, K. (2020). El consumo de información durante el confinamiento por coronavirus: medios, desinformación y virus. Barcelona:Digilab. Universitat Ramón Llull. Recuperado de http://www.digilab.cat/ disponible-el-informe-el-consumo-de-informaciondurante-el-confinamiento-por-el-coronavirus/

Molina-Cañabate, J. P., \& Magallón-Rosa, R. (2020). Desinformación y periodismo científico. El caso 
de Maldita Ciencia. Revista Mediterránea de Comunicación, 11(2). doi: 10.14198/ MEDCOM2020.11.2.4

Nielsen, R. K., Fletcher, R., Newman, N., Brennen, J., \& Howard, P. N. (2020). Navigating the 'infodemic': how people in six countries access and rate news and information about coronavirus. Reuters Institute. Recuperado de https://reutersinstitute.politics.ox.ac. uk/infodemic-how-people-six-countries-access-andrate-news-and-information-about-coronavirus

Nyhan, B., \& Reifler, J. (2012). Misinformation and factchecking: Research findings from social science. New America Foundation Media Policy Initiative Research Paper. Recuperado de https://www. newamerica.org/oti/policy-papers/misinformationand-fact-checkingl

Pennycook, G., McPhetres, J., Zhang, Y., \& Rand, D. G. (2020). Fighting COVID-19 misinformation on social media: Experimental evidence for a scalable accuracy nudge intervention. Preprint. doi: https://doi. org/10.31234/osf.io/uhbk9

Pennycook, G., Cannon, T., \& Rand, D. G. (2018). Prior Exposure Increases Perceived Accuracy of fake news. Journal of Experimental Psychology, 47(12), 1865-1880. doi: 10.1037/xge0000465

Reicher, S. (2020, 13 de mayo). Transparency is key in a crisis - so why isn't the British government being straight with us?. The Guardian. Recuperado de https://www.theguardian.com/commentisfree/2020/ may/13/british-people-lockdown-coronavirus-crisis

Seely, B. (2018). A Definition of Contemporary Russian Conflict: How Does the Kremlin Wage War? Henry Jackson Society. Recuperado de https:// henryjacksonsociety.org/publications/a-definition-ofcontemporary-russian-conflict-how-does-the-kremlinwage-war/

Shao, C., Ciampaglia, G., Varol, O., Kai-Cheng, Y., Flammini, A., \& Menczer, F. (2018). The spread of low-credibility content by social bots. Nature Communications, 9(1), 4787. doi: 10.1038/s41467018-06930-7

Tárdáguila, C., Benevenuto, F., \& Ortellado, P. (2018, 17 de octubre). Fake news is poisoning Brazilian politics. WhatsApp can stop it. The New York Times.
Recuperado de https://www.nytimes.com/2018/10/17/ opinion/brazil-election-fake-news-whatsapp.html.

Vallés, M. (1999). Técnicas cualitativas de investigación social. Reflexión metodológica y práctica profesional. Madrid, España: Síntesis.

Vázquez-Herrero, J., Vizoso, Á., \& López-García, X. (2019). Innovación tecnológica y comunicativa para combatir la desinformación: 135 experiencias para un cambio de rumbo. El Profesional de la Información, 28(3), e280301. doi: 10.3145/epi.2019.may.01

Vosoughi, S., Roy, D., \& Aral, S. (2018). The spread of true and false news online. Science, 359(6380), 1146-1151. doi: 10.1126/science.aap9559

Wardle, C., \& Derakhshan, H. (2017). Information Disorder: Toward an interdisciplinary framework for research and policy making. Council of Europe. Recuperado de https://rm.coe.int/ information-disorder-toward-an-interdisciplinaryframework-for-researc/168076277c

World Health Organization. (2020). Novel Coronavirus (2019-nCoV). Situation Report-13. World Health Organization. Recuperado de https:/l www.who.int/docs/default-source/coronaviruse/ situation-reports/20200202-sitrep-13-ncov-v3. pdf?sfvrsn=195f4010_6 